\title{
Memorial psychophysics for visual area: The effect of retention interval
}

\author{
SIMON KEMP \\ University of Canterbury, Christchurch, New Zealand
}

\begin{abstract}
Previous studies (e.g., Kerst \& Howard, 1978) have shown that remembered visual areas are related to actual areas by a more compressive power function than are perceived visual areas. Experiment 1 used a between-groups design to replicate this effect for a map of Europe and showed that compression increased as the retention interval, the time between map inspection and area estimation, increased from 2 min to 1 week. Experiment 2 obtained similar results with a similar design, except that a simple two-region map was used to reduce uncertainty. Overall, the results suggest that two processes may be operating: uncertainty and gradual transformation of the representation of the map in memory.
\end{abstract}

In a wide variety of circumstances, the estimated magnitude of a sensation or perception is related by a power function to the physical magnitude of the stimulus (S. S. Stevens, 1975). The existence of this relationship suggests that a similar function may relate the remembered magnitude of a stimulus to the physical magnitude. The possibility of such a relationship has been explored, in particular, in experiments requiring judgment of perceived and remembered physical area (Algom, Wolf, \& Bergman, 1985; Björkman, Lundberg, \& Tärnblom, 1960; Chew \& Richardson, 1980; Kerst \& Howard, 1978; Moyer, Bradley, Sorensen, Whiting, \& Mansfield, 1978). Two results have clearly emerged from this research. First, judgments of remembered area are related by a power function to the physical area. Second, the memorial exponent, the exponent of the power law function relating remembered to physical area, is smaller than the perceptual exponent, the exponent relating perceived to physical area. Over the studies listed, the former exponent ranged from 0.46 to 0.68 , and the latter ranged from 0.64 to 0.84 . Thus, all studies produced a compressive function (exponent of less than one) for perceived area, which was further compressed for remembered area.

There are a number of hypotheses that could be put forward to account for these results. In the first place, it could be that the lowered exponent in memorial conditions results from uncertainty. There are different versions of this hypothesis. Under that suggested by Kerst and Howard (1978), subjects in memorial conditions experience greater uncertainty than those in perceptual conditions and employ a more conservative range of area estimates that are closer to the standard area. Response

I am grateful to Clare Lange for help in conducting some of the experiments, to Phoebe Gray for help in coding, and to R. W. Proctor, P. N. Russell, W. M. Wiest, and two anonymous reviewers for their useful suggestions on earlier drafts of the manuscript. My mailing address is Simon Kemp, Department of Psychology, University of Canterbury, Christchurch, New Zealand. restriction of this kind also has been indicated in other magnitude estimate experiments (e.g., S. S. Stevens \& Greenbaum, 1966). Alternatively, as Algom et al. (1985) suggested following the lead of Teghtsoonian (1971), the effect of uncertainty may be to widen the effective stimulus range for each of the remembered areas. In either case, the effect of uncertainty would be to lower the value of the exponent in memorial conditions.

Kerst and Howard (1978) rejected the uncertainty hypothesis as inconsistent with the results of an experiment that required judgments of remembered and perceived distance. They found a perceptual exponent of 1.04 and a memorial exponent of 1.10 from their experiment, which indicated that the memorial exponent is not necessarily lower than the perceptual exponent, and hence that uncertainty is unlikely to be important in all judgments of remembered stimuli. It should be noted, however, that Kerst and Howard's result is not found in all distance estimation experiments: in a recent meta-analysis of 70 studies of distance estimation, Wiest and Bell (1985) found an average perceptual exponent of 1.08 and an average memorial exponent of 0.91 . Such results are what would be expected from uncertainty.

Alternatives to the uncertainty hypothesis suggest that the lowered memorial exponent results from transformation of the trace produced by the stimulus. Perhaps the most specific of these alternatives is the reperceptual hypothesis (Kerst \& Howard, 1978; Moyer et al., 1978). According to this hypothesis,

the physical stimulus arriving at the receptors is processed or transformed into a perceptual experience. This transformation yields the familiar power-function relation between physical magnitude and estimated psychological magnitude. This percept is then stored.... At recall a second re-perceptual transformation, very similar or identical to the initial perceptual transformation, is assumed to operate on the stored information... Thus two power functions intervene between the actual physical magnitude of an object and its memorial size estimate. (Kerst \& Howard, 1978, p. 331) 
This model predicts that the memorial exponent should equal the square of the perceptual exponent: for example, if the perceptual exponent were 0.8 , the memorial exponent should be $0.8^{2}$ or 0.64 . In fact, all the experiments performed so far using area as the relevant dimension have provided results in fairly good numerical agreement with this hypothesis.

Another, rather different, hypothesis is that the memory of a stimulus might gradually degrade or transform over time, and that the gradual transformation might utilize whatever perceptual process provides the initial perceptual transformation. Historically, this hypothesis might be traced to the theorizing of Koffka (1936), who suggested that Gestalt principles might be applied to mnemonic as well as perceptual processes. Indeed, there is empirical evidence that Gestalt principles do account for some of the distortions in the way maps are remembered. For example, A. Stevens and Coupe (1978) found that subjects tended to distort the geographical relation between two locations to conform in the direction of the relation between superordinate units (e.g., the Atlantic entrance of the Panama canal is judged to lie due east of the Pacific entrance). Tversky (1981) found the Gestalt principles of proximity and common fate to operate in the alignment and rotation of geographical entities.

In the present context, the gradual transformation hypothesis, as it might be termed, resembles the reperceptual hypothesis in supposing, first, that there is transformation of the percept after perception and, second, that the further transformation uses a perception-like process. Thus, if the initial perceptual transformation were compressive, we would expect further compression to occur in memorial estimation. The gradual transformation hypothesis differs from reperception, however, in that the transformation is supposed to be gradual and continuous. Although the gradual transformation hypothesis leads to less specific quantitative predictions than does the reperceptual hypothesis, there is empirical support for the hypothesis in that features of remembered pictures appear to be forgotten at different rates (e.g., Bartlett, 1932; Mandler \& Parker, 1976; Mandler \& Ritchey, 1977).

The following experiments required estimates of relative areas in perceptual and memorial conditions. They differed from previous research in that the retention interval, the length of time elapsing between seeing the stimulus and estimating its area, was varied in different memorial conditions. Although this interval has varied in previous research (e.g., $24 \mathrm{~h}$ in Moyer et al., 1978; $2 \mathrm{~min}$ in Kerst \& Howard, 1978), the effect of varying it has not been investigated systematically.

One reason for examining the effect of manipulating the retention interval is to decide between the hypotheses outlined above. If the reperceptual hypothesis provides the sole explanation of why the memorial exponent is less than the perceptual one, then varying the length of time between seeing the stimulus and estimating its area should not affect the value of the memorial exponent. On the other hand, both the uncertainty and the gradual trans- formation hypotheses predict that the value of the exponent should decrease as the retention interval is increased. For the uncertainty hypothesis, this should occur because subjects should become more uncertain with increased time after viewing the stimulus, whereas under the gradual transformation hypothesis, the exponent should be progressively lowered as the trace of the stimulus is altered by the continuous gradual application of the perceptual process.

\section{EXPERIMENT 1}

\section{Method}

Subjects. One hundred fifty-five subjects, all first-year students in the department of psychology, participated in the experiment.

Materials. Outline maps $(21.0 \times 29.7 \mathrm{~cm})$ of the European continent and the Mediterranean (equal area projection) were provided for four of the five experimental groups. The maps showed only the boundaries and names of 25 European countries and islands. The subjects recorded their estimates of the areas of the countries and islands on answer sheets containing the 25 names in different random orders. Instruction sheets were also provided.

Procedure. The subjects were divided into five experimental groups. The subjects in the perceptual group had maps as they made their area estimates. The subjects in the three memory groups were given maps and instructed to "study the maps so that [they could] visualize them later." They were further told to pay particular attention to the relative sizes or areas of the different countries and islands. Ten minutes were allowed for studying the maps. Subjects in the first memory group estimated areas $2 \mathrm{~min}$ after the maps had been removed, during which time the answer sheets were distributed; subjects in the second memory group estimated areas 90 min after map removal; and subjects in the third memory group estimated areas 7 days after map removal. A fifth, control, group performed area estimation without previously viewing the maps.

All subjects, when given their answer sheets, were instructed as follows:

Please rate the following European countries and islands in terms of their geographical size or area. Assume the United Kingdom is 100 units in size. If you think a country is twice as large as that, rate it 200 units. If you think it a quarter as large, rate it 25 units. Your ratings do not have to be in round numbers; feel free to use any number "in between" that you feel describes the size of the country or island. Please record your answer alongside the country or island. Please give a rating to each area even if you really have no idea how large it is or even if you have never heard of it before.

No time limit was set for the completion of the task; all subjects completed it in $30 \mathrm{~min}$, most within $20 \mathrm{~min}$.

\section{Results and Discussion}

The geometric mean of the area estimates was obtained for each country and island by averaging across all subjects in each group. Figure 1 presents the logarithm of these mean estimates plotted against the logarithm of the actual area. The exponent of the power function relating the estimated to the actual area was extracted as the slope of the least squares regression line. The figure shows the exponents and the values of the correlation coefficients, which provide a measure of the goodness of fit of the power functions. The results shown in the figure suggest that, for the perceptual and memory groups, the power 


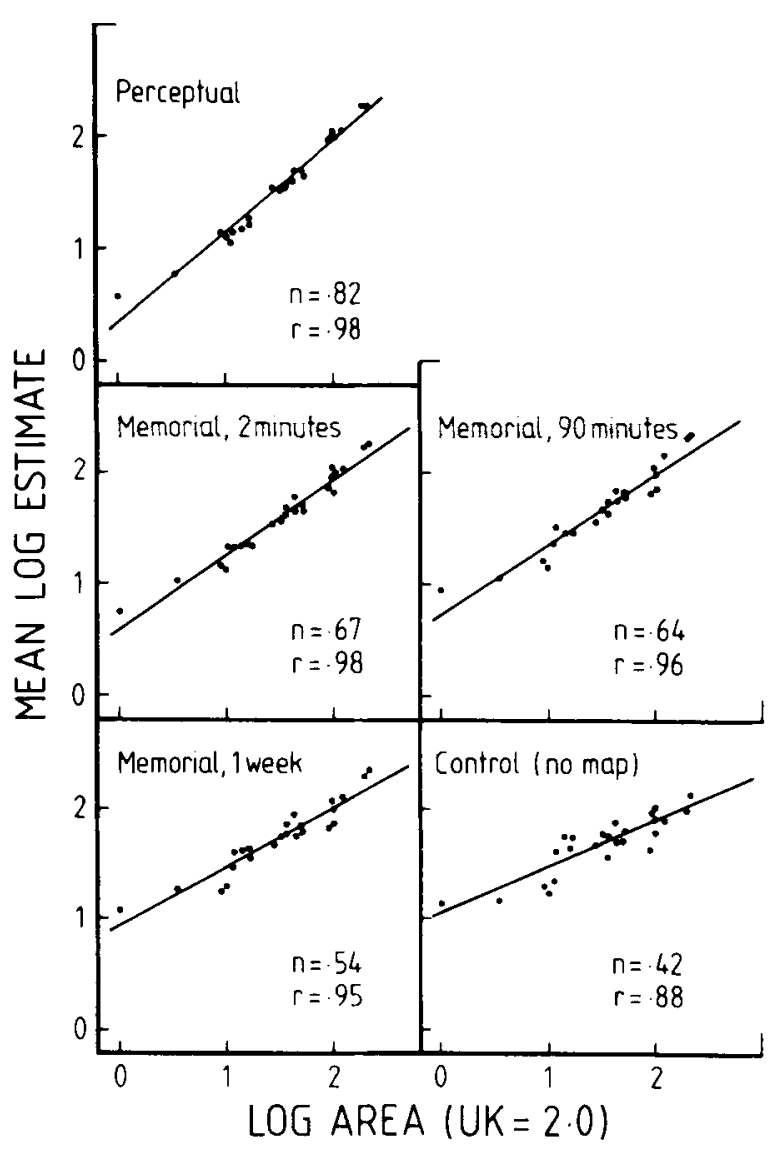

Figure 1. Log geometric mean estimates of the areas of European countries and islands as a function of actual area (referenced to the United Kingdom, equal to $\mathbf{1 0 0}$ units) for five experimental conditions in Experiment 1. Linear regression lines have been drawn, and slopes $(n)$ and correlation coeficients $(r)$ are shown.

function is a reasonably good fit, and that the exponent decreased from perceptual to memory conditions and decreased as the interval between map inspection and estimation increased.

Analysis of the individual data also was undertaken in order to apply tests of statistical significance. Exponents and correlation coefficients were calculated for each subject by logging each of the subject's 25 estimates and regressing these on the logged actual areas, to obtain slope and Pearson product-moment correlation estimates. A few subjects gave fewer than 25 estimates; in these cases, the exponent and correlation coefficients were calculated from only those areas they had estimated. (A parallel analysis that was performed, omitting these subjects entirely, gave substantially the same results.)

Table 1 shows the average values of exponent and correlation coefficient obtained in this way. Comparison of these values with those obtained from the group analysis indicates that the exponents obtained from the two analyses are very similar, but that the correlation coefficients obtained from the individual analyses are generally lower and more sensitive to experimental conditions than those obtained from the group analysis. A similar trend may also be seen in the results reported by Kerst and Howard (1978, Tables 1 and 2).

Analyses of variance (ANOVAs) of the individual data showed a significant difference in exponent over the five conditions $[F(4,150)=17.5, p<.05, M S e=.029]$. A priori contrasts showed significant differences between the perceptual and the three memory groups $[t(150)=$ $5.46, p<.05$ ], between the memory groups and the control gruop $[t(150)=4.25, p<.05]$, and between the 2 $\min$ and 1 -week memory groups $[t(150)=3.49$, $p<.05, \mathrm{MSe}=.015]$. A similar analysis of the correlation coefficients showed a significant effect over the five conditions $[F(4,150)=20.0, p<.05$, MSe $=.015]$. A priori contrasts showed significant differences between the perceptual and the three memory groups $[t(150)=$ $5.41, p<.05]$, between the memory groups and the control group $[t(150)=4.92, p<.05]$, and between the 2$\min$ and 1 -week memory groups $[t(150)=4.10$, $p<.05]$. The exponents obtained by the memory groups, particularly by the 2-min memory group, are close to the square of that obtained from the perceptual group (.67), as predicted by the reperceptual hypothesis. On the other hand, the decrease in the memorial exponent with increased time between seeing the map and estimating the areas is consistent with both the gradual transformation and the uncertainty hypotheses, but is not predicted by reperception alone.

The values of the correlation coefficients in Table 1 and Figure 1 show a progressive decrease from perceptual to memorial conditions and as the interval between viewing and area estimation was increased. Such a result indicates that, over time, responses became more varied and more inaccurate, and suggests that there was at least some effect of uncertainty in the results. However, uncertainty may not have been the only cause of the lowered memorial exponent in this experiment. It could be, for example, that as the retention interval was lengthened, there was both increased uncertainty and gradual transformation of the memorial trace.

It seems likely in the present experiment, as well as in those of Chew and Richardson (1980), Kerst and Howard (1978), and Moyer et al. (1978), that uncertainty might be created by the rather complex stimuli used. In Experiment 1 , for example, the effective stimulus range might be enhanced because of the large number of areas to be estimated. Hence, the next experiment used only two areas and called for only one area estimate, of the larger area in relation to the smaller. With such a stimulus, the ef-

Table 1

Mean Exponent and Correlation (Averaged Over Individual Subjects) for Each Experimental Condition of Experiment 1

\begin{tabular}{lccc}
\hline \multicolumn{1}{c}{ Condition } & Exponent & Correlation & $n$ \\
\hline Perceptual & .82 & .95 & 27 \\
Memorial-2 min & .67 & .87 & 41 \\
Memorial-90 min & .65 & .81 & 33 \\
Memorial-1 week & .54 & .76 & 38 \\
Control & .43 & .65 & 16 \\
\hline
\end{tabular}


fects of uncertainty should be greatly reduced, if not eliminated, because there is considerably less to be remembered. Such an experiment permits a choice between the uncertainty hypothesis and the gradual transformation hypothesis. Because uncertainty is minimized, the uncertainty hypothesis predicts that there should be little difference in estimated area between the perceptual and memorial conditions or within the memorial conditions as the time interval between vision and estimation is lengthened. On the other hand, the gradual transformation hypothesis predicts that the estimates of the larger area should decrease between perceptual and memorial conditions and as the retention interval is increased.

\section{EXPERIMENT 2}

\section{Method}

Subjects. Three hundred ninety-six subjects, all first-year students in the department of psychology, participated. None had participated in Experiment 1.

Stimulus. A slide was prepared that featured a small, irregular yellow tongue on a red background. The relative area of yellow:red was 100:1511. The slide, which is illustrated in black and white in Figure 2, was projected onto a screen via a Kodak Carousel slide projector in a laboratory area.

A brief preexperiment was conducted with this and five similar slides showing different proportions of yellow:red. This preexperiment found a compressive function with an exponent of 0.87 to relate perceived to actual areas. The purpose of the preexperiment was to establish that relative underestimation of the yellow area in the experiment proper might be interpreted in terms of compression rather than in terms of some other mechanism producing underestimation.

Procedure. The subjects were run in groups of about 30 , and performed under one of four conditions. The subjects in the perceptual condition were asked to estimate the area of red relative to that of the yellow tongue, which they were told had an area of 100 units. The slide was then shown for $15 \mathrm{sec}$. The subjects in the three memorial conditions were asked to form a mental picture of the slide that they could later recall, and the slide was shown for $15 \mathrm{sec}$. The three memorial groups were asked $2 \mathrm{~min}, 90 \mathrm{~min}$, or 1 week later to recall and visualize the slide previously shown, and were reminded that it had featured an irregular yellow tongue

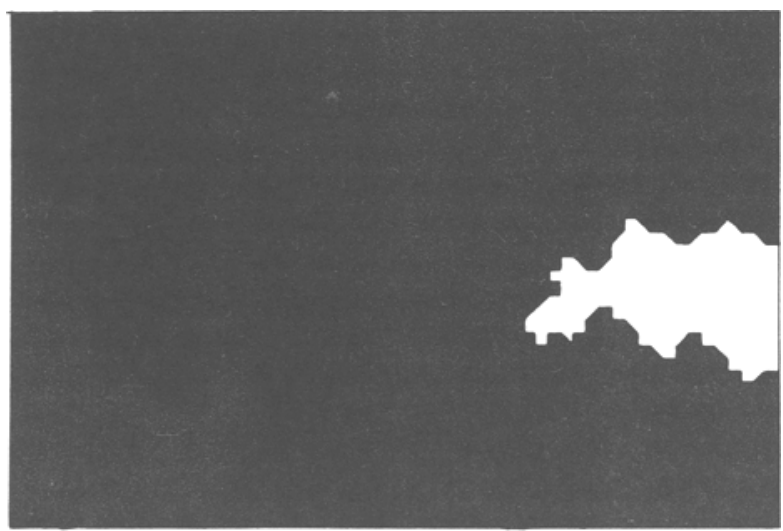

Figure 2. Black-and-white illustration of the stimulus presented in Experiment 2. The dark area was red and the light area was yellow in the actual stimulus.
Table 2

Geometric Mean of the Estimates of the Red Area and Corresponding Area Exponent for Each Condition of Experiment 2

\begin{tabular}{lccccr}
\hline \multicolumn{1}{c}{ Condition } & $\begin{array}{c}\text { Geometric } \\
\text { Mean }\end{array}$ & \multicolumn{3}{c}{$M(\log$} & \multicolumn{2}{c}{$S D$ (Log } \\
\hline Perceptual & 918 & .82 & 2.96 & 0.13 & 85 \\
Memorial-2 min & 706 & .72 & 2.85 & 0.28 & 82 \\
Memorial-90 min & 654 & .69 & 2.82 & 0.24 & 115 \\
Memorial-1 week & 479 & .58 & 2.68 & 0.28 & 114 \\
\hline
\end{tabular}

Note-The means and standard deviations of the logarithms of the estimates and the numbers of subjects $(n)$ in each condition are also shown.

on a red background. They were then asked to estimate the area of red relative to that of the yellow tongue (100 units).

The subjects in the memorial groups were not told that they were to make area judgments, and precautions were taken to prevent their finding out from other groups. Two other slides featuring a dot display and a crowd scene were also shown to the subjects immediately following the area slide to prevent conscious rehearsal of the slide's features.

\section{Results}

Table 2 shows the geometric means of the red area estimates obtained in each of the four conditions. The table also shows the exponents of the power function relating estimated to actual area. In this experiment, the exponents were found by exact calculation, rather than by statistical process, by dividing the logged mean area estimates by the logged actual area of red. [According to the power law, estimated ratio of red to yellow $=$ (actual ratio of red to yellow) ${ }^{n}$.] An ANOVA on individual logged area estimates showed a significant effect of conditions $[F(3,392)=22.3, p<.01, M S e=.060]$. A priori contrasts showed a significant difference between the perceptual and the three memory conditions $[t(392)=6.03$, $p<.01]$ and between the 2-min and 1-week memorial conditions $[t(392)=4.75, p<.01]$.

These results show that, in a situation in which the opportunity for uncertainty was experimentally minimized by reducing the stimulus range presented to only two different areas, a standard and a comparison, the important results of Experiment 1 were replicated. Area estimates and exponents were smaller in the memorial conditions than in the perceptual one. Moreover, it is clear that the red area estimates and exponents decreased as the retention interval increased. The results thus support the gradual transformation hypothesis.

Additional support for this hypothesis is found in the results relating to the variability of the area estimates. The standard deviations of the logarithms of the area estimates presented in Table 2 are similar over the three memorial conditions. This similarity indicates that the effect of lengthening the retention interval in this experiment cannot be explained by increased uncertainty.

\section{GENERAL DISCUSSION}

Overall, the present experiments suggest that more than one mechanism may be operating in memorial psychophysics experiments. Some effect of uncertainty is sug- 
gested by the fact that memorial, but not perceptual, exponents were lower in Experiment 1 than Experiment 2 and by the change in the individual correlation coefficients with experimental conditions found in Experiment 1. Neither reperception nor gradual transformation explains the latter result very well. If, for example, individuals varied in their rates of gradual transformation, one might expect the group results to show more variation about the regression line as the time after map inspection increased, but the intraindividual results should not show such variation. The pattern of results obtained, in fact, seems most easily explained by the hypothesis that subjects were affected by uncertainty and that this uncertainty was more pronounced for some countries and islands than for others.

Experiment 2 shows that results similar to those of Experiment 1 can be obtained when the opportunity for uncertainty is reduced by experimental means, and hence that uncertainty is not the only mechanism responsible for the lowered memorial exponent. Furthermore, although the results of Experiment 1 could be explained by the combined operation of uncertainty and reperception, such an explanation fails for Experiment 2, because no effect of lengthening the retention interval would be expected in this case. The results of this experiment are predictable by only one of the hypotheses considered here, that of gradual transformation. In general, however, it seems probable that a mixture of uncertainty and gradual transformation might operate when area judgments of remembered visual stimuli are made.

Acceptance of the gradual transformation hypothesis is not without theoretical problems, in that it is unclear what sort of gradual transformation is taking place. Also, it is not clear exactly what is being transformed. Obviously important here is the issue of whether the area information on the stimulus maps is encoded and stored in memory in a propositional or analog (picture-like) form. This issue, in turn, relates to the much-debated question of whether mental imagery in general arises from propositional or analogical processes (see, e.g., Anderson, 1978; Finke, 1985; Kosslyn, 1980; Pylyshyn, 1973, 1984; Shepard, 1978).

At first glance, the gradual transformation hypothesis and the results of the experiments reported here seem to support the idea that the stimulus is represented in memory in an analogical, picture-like way, as suggested, for example, by Finke (1985), Kosslyn, (1980), and Shepard (1978). Otherwise, why should the memory trace be apparently subject to a perception-like process? Further consideration, however, suggests that the question is not so easily resolved. As Pylyshyn (1973) pointed out, the analogical model is that of an observer regarding a picture: "The whole vocabulary of imagery uses a language appropriate for describing pictures and the process of perceiving pictures. We speak of clarity and vividness of images, of scanning images, of seeing new patterns in images ..." (p. 8). The present research, however, suggests that the image, analogous to the picture, is continuously transforming; smaller areas gradually become relatively larger while larger areas become relatively smaller. Although such a process is not incompatible with the analogic model, it is certainly not explained by it. Moreover, although it is true that real-life scenes may be blurred or rendered indistinct by, say, poor lighting, the relative areas of objects in the real world or surfaces shown in a picture generally remain constant, in the latter case often even if the viewing angle is changed.

The propositional account of imagery also does not provide an explanation of gradual transformation. It is true that areas or "magnitudes can easily be represented in a propositional model as arguments of propositions" (Anderson, 1978, p. 261), and clearly these arguments could be randomly changed or perhaps altogether lost by a process of forgetting. There is, however, no necessary reason why the values of such arguments should gradually change so as to become more like each other, as is suggested by the results reported here. Thus the process of gradual transformation suggested in this paper is compatible with either an analog or a propositional model, but is not predicted by either of them.

A final issue concerns the generality of the phenomenon reported. Are other remembered dimensions subject to gradual transformation in the same way as is area? There is clear experimental evidence that perceptual distortion is not always amplified in memory; for example, circles that contain gaps do not progressively close up with elapsed time to recall (Holmes, 1968). Results reported by Kerst and Howard (1978) indicate that the memorial exponent is not always lower than the perceptual one for the dimensions of brightness and loudness, and Osaka (1987) recently reported that the remembered exponent $(0.52)$ for pyridine smell is significantly greater than the perceptual one (0.38). Preliminary data from my own laboratory suggest that numerosity judgments are generally subject to gradual transformation in the same way as area estimation, but there are some apparent discrepancies. For example, remembered estimates of the number of dots seen progressively declined with increasing retention interval, whereas judgments of the number of dots "seen" in the image did not. Hence, overall, in light of the research conducted, it would seem unwise to expect gradual transformation to occur or even to predict whether the memorial exponent will be larger or smaller than the perceptual one, for stimulus dimensions other than area.

\section{REFERENCES}

Algom, D., Wolf, Y., \& Brgman, B. (1985). Integration of stimulus dimensions in perception and memory: Composition rules and psychophysical relations. Journal of Experimental Psychology: General, 114, 451-471.

ANDERSON, J. R. (1978). Arguments concerning representations for mental imagery. Psychological Review, 85, 249-277.

BARTLETT, F. C. (1932). Remembering: A study in experimental and social psychology. Cambridge, England: Cambridge University Press.

BJörkman, M., LunDBERG, I., TÄrnbLOM, S. (1960). On the relationship between percept and memory: A psychophysical approach. Scandinavian Journal of Psychology, 1, 136-144.

Chew, E. I., Richardoson, J. T. E. (1980). The relationship between 
perceptual and memorial psychophysics. Bulletin of the Psychonomic Society, 16, 25-26.

Finke, R. A. (1985). Theories relating mental imagery to perception. Psychological Bulletin, 98, 236-259.

Holmes, D. S. (1968). Search for "closure" in a visually perceived pattern. Psychological Bulletin, 70, 296-312.

Kerst, S. M., \& HowaRD, J. H. (1978). Memory psychophysics for visual area and length. Memory \& Cognition, 6, 327-335.

KoffkA, K. (1936). Principles of Gestalt Psychology. London: Kegan Paul.

KossLyn, S. M. (1980). Image and mind. Cambridge, MA: Harvard University Press.

Mandler, J. M., Parker, R. E. (1976). Memory for descriptive and spatial information in complex pictures. Journal of Experimental Psychology: Human Leaming \& Memory, 2, 38-48.

MANDleR, J. M., \& RitcheY, G. H. (1977). Long-term memory for pictures. Jourmal of Experimental Psychology: Human Learning \& Memory, 3, 386-396.

Moyer, R. S., Bradley, D. R., Sorenson, M. H., Whiting, J. C., Mansfield, D. P. (1978). Psychophysical functions for perceived and remembered size. Science, 200, 330-332.

OsAKA, N. (1987). Memory psychophysics for pyridine smell scale. Bulletin of the Psychonomic Society, 25, 56-57.
Pylyshyn, Z. W. (1973). What the mind's eye tells the mind's brain: A critique of mental imagery. Psychological Bulletin, 80, 1-24.

Pylyshyn, Z. W. (1984). Computation and cognition: Toward a foundation for cognitive science. Cambridge, MA: MIT Press.

ShepARD, R. N. (1978). The mental image. American Psychologist, 33, 125-137.

Stevens, A., \& Coupe, P. (1978). Distortions in judged spatial relations. Cognitive Psychology, 10, 422-437.

Stevens, S. S. (1975). Psychophysics: Introduction to its perceptual, neural and social prospects. New York: Wiley.

Stevens, S. S., Greenbaum, H. B. (1966). Regression effect in psychophysical judgment. Perception \& Psychophysics, 1, 439-446.

Teghtsoonian, R. (1971). On the exponents in Stevens's law and the constant in Ekman's law. Psychological Review, 78, 71-80.

Tversky, B. (1981). Distortions in memory for maps. Cognitive Psychology, 13, 407-433.

Wiest, W. M., \& Bell, B. (1985). Stevens's exponent for psychophysical scaling of perceived, remembered, and inferred distance. Psychological Bulletin, 98, 457-470.

(Manuscript received March 9, 1987; revision accepted for publication January 11,1988 .) 